\title{
Fine structural spectrometry and spectroradiometry of combustion products of energy fuels
}

\author{
$N I$ Moskalenko, $Y u$ A Ilyin, $O V$ Zotov, $I R$ Dodov* and $A R$ Akhmetshin \\ Kazan State Power Engineering University, Russia, 420066, Kazan, st.Krasnoselskaya 51
}

\begin{abstract}
The results of experimental studies of the fine structure of the spectra of molecular absorption and emission of combustion products of energetic fuels and their application in solving problems of radiative heat transfer in structurally inhomogeneous multicomponent media are considered. The methods of determining the parameters of the spectral absorption lines from the experimental emission spectra of the flame in the combustion products of gas fuel the identification of optically active ingredients in the combustion products and anthropogenic emissions into the atmosphere are analyzed. Attention is drawn to the fact that the emission of gas components is selective and the methods for calculating the radiative heat exchange in the combustion chambers of power units must take into account the acute selection of the molecular absorption spectra of radiation.
\end{abstract}

\section{Introduction}

Solving problems of radiative heat transfer in the combustion chambers of power units requires knowledge of the radiation characteristics of the optically active ingredients of the gas and dispersed phase of the combustion products. For the gas phase of the combustion products the main radiation characteristic is the spectral transmittance function (STF) which depends on temperature in a complex way and is determined by the fine structure of the absorption (emission) spectra of the combustion products. Strict solutions of the problems of radiative heat transfer in a structurally inhomogeneous medium require taking into account the acute selection of absorption spectra and its dependence of the temperature of the ingredients of the gas phase of the combustion products. Thus the gaseous medium is a selective source of radiation and STF for a medium that is not uniform in temperature and ingredient composition can be calculated by numerical modeling using hightemperature atlases of the parameters of the spectral absorption lines obtained by complex application of the theory of spectral analysis of vibrational-rotational spectra. Below are considered measuring complexes of high spectral resolution the results of experimental studies and their application for solving problems of radiative heat exchange and determining the ingredient composition of combustion products of energy fuels and atmospheric anthropogenic emissions.

\section{Spectral installation high-temperature gas radiator SUVGI-2}

The flame measuring complex of SUVGI-1 described in [1] has a drawback: the inhomogeneity of the temperature of the gas medium at the boundaries of the flame zones. This deficiency was eliminated in the flaming measuring complex of high spectral resolution SUVGI-2 described below. On the working layouts of this installation and the experimental sample a large part of the spectral measurements were taken which form the basis of the parametrization of the radiative characteristics of the gas components of the combustion products [1].

The developed SUVGI-2 complex is intended for recording flame absorption spectra and for recording flame emission spectra at high spectral resolution under controlled conditions and has full metrological support for the "block diagram" of the measuring complex described in [2]. The spectral complex functions in the spectral range of 0.2-100 microns. The spectra are recorded using an FS-1 Fourier spectrometer and an SDL-1 spectrometer. When operating in the registration mode of absolute flame emission spectra calibration radiation sources are used. Fig. 1 shows the optical layout of the SUVGI-2 installation in the measurement version of the absorption spectra of radiation by a flame of a radiation source which is used as a globar belt tungsten lamp or a DDS-30 deuterium lamp.

Radiation from source 1 is transmitted via a matching optical attachment with mirrors 2.3 through the input window 4 (1) to the entrance slit of a multi-way cell (MWC) and after multiple reflections from mirrors 5 (1), 5 (2), 6 through the output slit and the output window 4 (2) of the output optical set-top box with mirrors $7,8,9$ is projected onto the entrance slit of the spectrometer 10 . Mirrors 5 (1), 5 (2), 6 for protection against overheating by flame radiation are cooled with water.

The high-temperature flame emitter constructively represents the gas emitter unit which is placed over the

\footnotetext{
* Corresponding author: utisey@gmail.com
} 


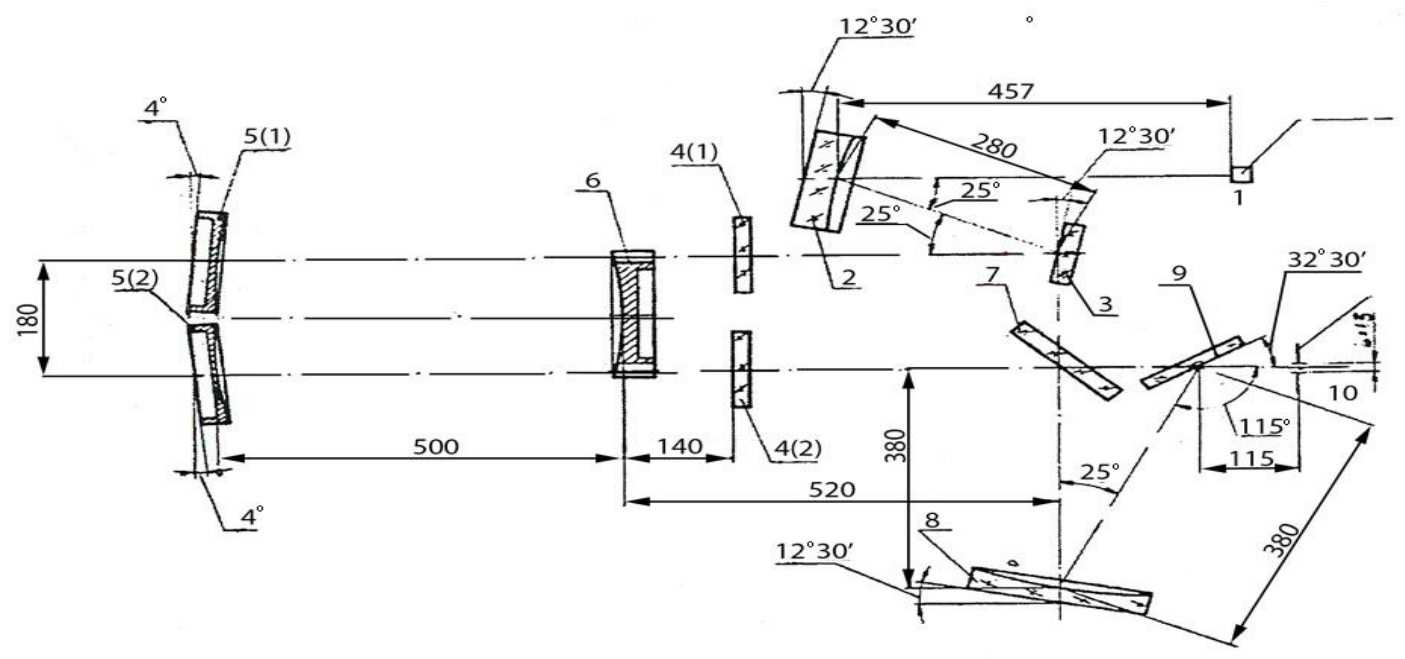

Fig. 1. Optical scheme of installation SUVGI-2.

optical bench and forms the flame zone between the mirrors 5 (1), 5 (2), 6 (Fig. 1), closed on top with a water-cooled cap with two protective windows. Flames formed during combustion of gases have a rectangular shape with a base size of $40 \times 20 \mathrm{~cm} 2$. The design of the burner allows to explore the hydrogen-oxygen hydrogenair and hydrocarbon flames. Measurements have shown that temperature inhomogeneity within the field of view of the optical system is $3 \%$. Various variants of optical schemes that are compatible with the system of multiple transmission of radiation built according to the White scheme make it possible to investigate the emission spectra of flames and the absorption spectra of continuous measurement by the flame in the optical path length range of $0.2 \div 16 \mathrm{~m}$. The flame temperature is measured by the method of self-reversal of spectral lines of water vapor of 1.38 and $1.87 \mu \mathrm{m}$ bands. The average relative error of flame temperature measurement is \pm $2 \%$. Measurement of gas flow rates was carried out with specially calibrated RS-5 rotameters. According to the ratio of the mass fractions of fuel and oxidizer the chemical composition of the combustion products is determined by thermodynamic calculation according to [3]. For absolute calibration of radiation spectra SIRSh 8.5-200-1 spectrometer lamps and Globar GMM previously calibrated by metrological standards provided are used. Fig. 2 illustrates the appearance of the measuring complex.

Measurements of emission spectra and absorption spectra of radiation by a flame allow us to determine the spectral nonequilibrium coefficients in flames [4] and the high spectral resolution with a known apparatus function of the spectrometer makes it possible to determine the parameters of spectral lines: intensity halfwidths and center positions.

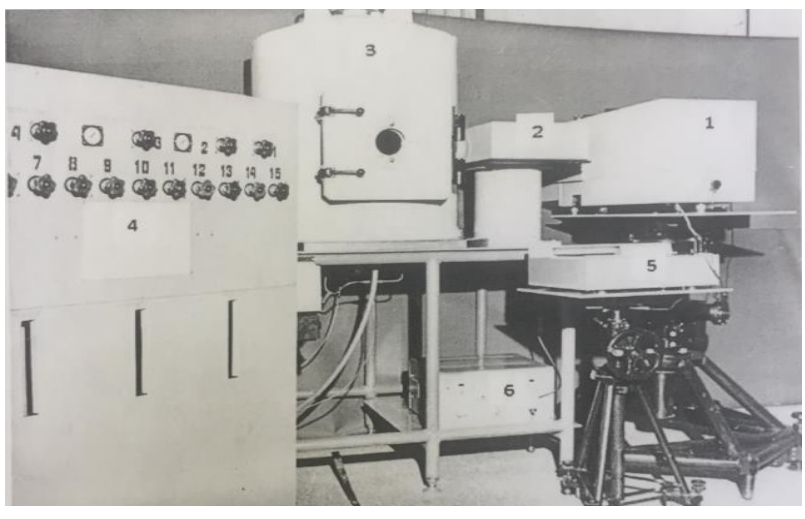

Fig. 2. Spectral installation of high-temperature gas emitter: 1 Fourier - high resolution spectrometer; 2 - matching optical settop box; 3 - high-temperature gas emitter; with multi-pass optical bench; 4 - vacuum-dosing system for supplying oxidizer and combustion products; 5 - grater; 6 - power supply.

For this the optical thickness spectrum is divided by the method of differential moments into separate contours of spectral lines which are then restored to the influence of the apparatus function of the spectrometer [1]. In [6] the use of a unique Fourier spectrometer UFS2 with a spectral resolution limit $\Delta=0.005 \mathrm{~cm}^{-1}$ in the spectrometry of combustion products was considered and to determine the parameters of weak lines of water vapor it was proposed to use the Sun as a source of radiation. Solar radiation is absorbed by atmospheric water vapor the altitude distribution of which is determined by radio sounding from a nearby weather station. The parameters of the spectral lines are determined by the nonlinear least squares method of measurement errors $[5,6]$ taking into account the effects of the apparatus function of the spectrometer for a structurally inhomogeneous atmosphere.

Solar radiation transmitted through the atmosphere is projected by movable mirrors onto the entrance slit of the spectrometer and is controlled in digital form for 
subsequent processing on a computer. In fig. 3,4 shows spectrograms of solar radiation for the spectral regions $6530-6600 \mathrm{~cm}^{-1}$ and $4990-5030 \mathrm{~cm}^{-1}$.

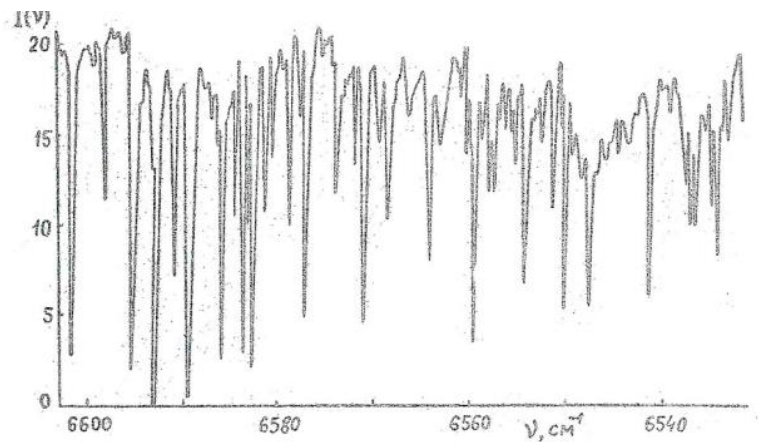

Fig. 3. Spectrograms of solar radiation for parts of the spectrum $6530-6600 \mathrm{~cm}^{-1}$.

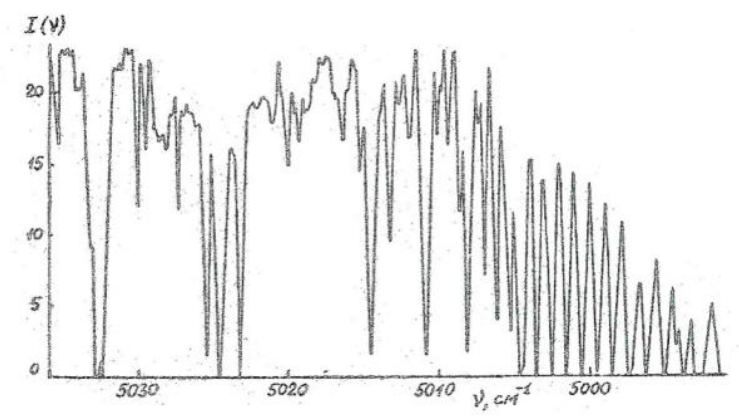

Fig. 4. Spectrograms of solar radiation for parts of the spectrum 4990-5030 $\mathrm{cm}^{-1}$.

The fine-structure spectrometry method was used to measure the composition of the flame zones of combustion of energy fuels in combustion chambers and in atmospheric anthropogenic emissions [7 - 9]. The high spectral resolution makes it possible to determine the spectral dependence of the attenuation of radiation by the dispersed phase of combustion products in the spectral region of $0.2-100 \mu \mathrm{m}$ which makes it possible to reveal the microstructure of sol particles of known chemical composition [10].

\section{Non-equilibrium gaseous emitter}

In fig. 5 shows a diagram of the design of a nonequilibrium gaseous emitter which allows the optical method to model structurally inhomogeneous media.

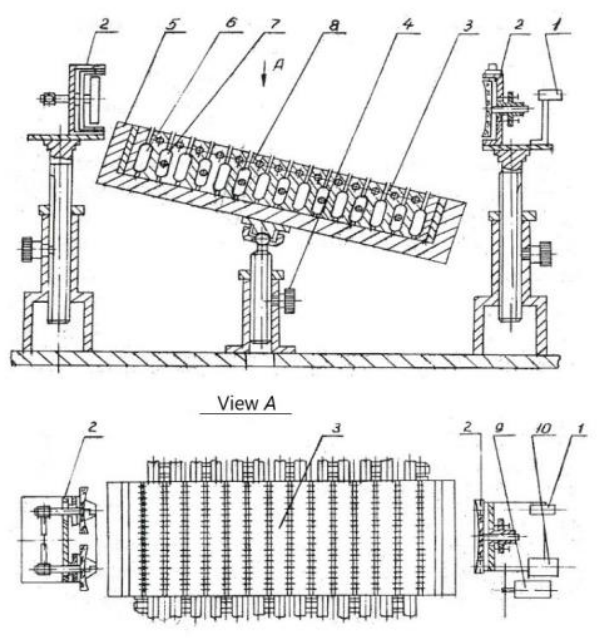

Fig. 5. . Diagram of the opto-mechanical part of the inhomogeneous gas emitter, side and top view: 1 - entrance slit; 2 - white's optical bench mirrors; 3 - multi-pass gas burner unit; 4 - rack; 5 - radiator base; 6,7 - capillaries for a combustible oxidizer; 8 - radiators; 9 - mechanical modulator radiation interrupter; 10 - spectrometer.

The non-equilibrium gaseous emitter is designed to investigate the transfer of radiation to non-equilibrium onto temperature and ingredient composition of combustion fuels of energy fuels. Non-equilibrium media are created using the multi-row emitter and White's multi-pass optical system by rotating it relative to the vertical plane of the flow of combustion products and changing the height of the plane of the White's optical system relative to the base of the bench. Temperature profiles depending on the localization of the mirrors Fig. 6, a are shown in Fig. 6, b, c, d, f.

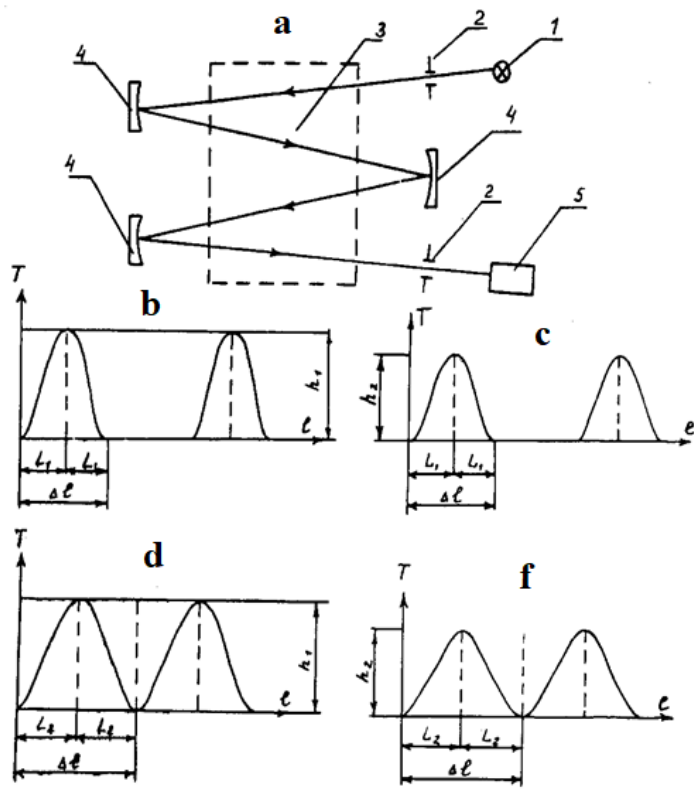

Fig. 6. Formation of temperature profiles for cases of fourfold passage of radiation through a flame and the corresponding temperature profiles $\mathrm{T}$ in the path of optical radiation: $1-$ radiation source; 2 - entrance and exit slits; 3 - flame zone; 4 mirrors; 5 - spectrometer. 


\section{References}

1. N.I. Moskalenko, S.O. Mirumyants, N.F. Loktev, R.Sh. Misbakhov, Equilibrium and non-equilibrium radiation processes: high-temperature environments, radiative heat transfer ed KGEU, 264 (2014)

2. N.I. Moskalenko, I.R. Dodov, Fine structured spectroscopy: measuring experimental studies and their applications, World chemistry forum 2019 theme: analysis, catalysis, nanoscience, 52 (2019)

3. V.E. Alemasov, Thermodynamic and thermophysical properties of combustion products, 490 (1972)

4. N.I. Moskalenko, A.V. Zaripov, Yu.A. Ilyin, Investigation of nonequilibrium hydroxyl emission spectra, Russian physics journals, 53(2), 107-113 (2010)

5. O.V. Zotov, A spectral setup for the study of atmospheric gases with a Fourier spectrometer of high resolution UFS-02, Transactions of the VII-th all-union symposium on molecular spectroscopy of high and super high resolution, 2, 277-229 (1986)

6. O.V. Zotov, Measurement of high-discharged solar IR absorption spectra of the atmosphere along oblique paths, Transactions of the VII-th all-union symposium on molecular spectroscopy of high and super high resolution, 2,93-97 (1986)

7. N.I. Moskalenko, Radiative characteristics of a hydrogen-oxygen flame, Journal of applied spectroscopy, 3, 407-415 (2010)

8. N.I. Moskalenko, R.Sh. Misbakhov, I.Z. Bagautdinov, N.F. Loktev, I.R. Dodov, Determination of ingredient composition of atmospheric emissions of the turbojet engine combustion gases by the fine-structure spectroscopy, Russian aeronautics, 59(3), 419-425 (2016)

9. N.I. Moskalenko, Identification of ingredients and determination of the composition of atmospheric emissions and products of combustion by finestructure spectrometry, Alternative energy and ecology, 6, 89-98 (2019)

10. N.I. Moskalenko, M.S. Khamidullina, Ya.S. Safiullina, I.R. Dodov, Study of spectral optical characteristics and microstructure of anthropogenic sols, Atmospheric and oceanic optics, 35(8), 628633 (2018)

11. Alberti, Validation of HITEMP-2010 for carbon dioxide at low temperatures and atmospheric pressures of 450-7600 cm-1 spectral range, Journal of quantitative spectroscopy and radiative transfer, 157, 14-33 (2015)

12. V.E. Alemasov, Mathematical modeling of hightemperature processes in power-generating installations, 256 (1989)

13. V.M. Gureev, Technological possibilities of increasing the efficiency of power plants based on a gas turbine engine, Izvestia vuzov Aviation technology, 2, 57-60 (2013)

14. L.S. Rothman, The HITRAN 2012 molecular spectroscopic database, Journal of quantitative spectroscopy and radiative transfer, 130, 4-50 (2013)

15. I.E. Gordon, The HITRAN2016 molecular spectroscopic database, Journal of quantitative spectroscopy and radiative transfer, 203, 3-69 (2017) 